\title{
$\beta$-Eudesmol (a Main Component of Atractylodes lancea)-Induced Potentiation of Depolarizing Neuromuscular Blockade in Diaphragm Muscles of Normal and Diabetic Mice
}

\author{
Masashi MUROI, Katsunao TANAKA, Ikuko KIMURA \\ and Masayasu KIMURA \\ Department of Chemical Pharmacology. Faculty of Pharmaceutical Sciences, \\ Toyama Medical and Pharmaceutical University. Toyama 930-01, Japan \\ Accepted February 16, 1989
}

\begin{abstract}
Potentiating effects of $\beta$-eudesmol on neuromuscular blockade were investigated in phrenic nerve-diaphragm muscles of normal and alloxan-diabetic mice. Pretreatment with $\beta$-eudesmol potentiated the blocking effects of succinylcholine to a greater extent in diabetic muscles than in normal ones. This effect was saturated after 30 -min pretreatment in normal muscles. Further potentiation was observed after 60 -min pretreatment in diabetic muscles. These results suggest that $\beta$-eudesmol modifies the sensitivity of diabetic mice to depolarizing blockers so that they have a higher susceptibility to these compounds.
\end{abstract}

$\beta$-Eudesmol is a naturally occurring compound from Atractylodes lancea (Sōzyutu), a traditional Sino-Japanese medicine. We previously reported that $\beta$-eudesmol is mainly responsible for the relaxing action of Keishika-zyutubu-tō (traditional Sino-Japanese prescription. Hozzai) in the neuromuscular junction in situ of diabetic mice. The blocking effect was 5.2-fold more potent in diabetic muscles than in normal ones (1). Succinylcholine (SuCh) was also more effective on the blocking action in diabetic (alloxan-, streptozotocin-, KK-CA ${ }^{y}-$ ) muscles, in contrast to d-tubocurarine by which the same extent of responses were observed in both kinds of isolated muscies (Ref. 2 and I. Kimura et al., unpublished observation). Furthermore, in this paper, we investigated the interaction between $\beta$-eudesmol and depolarizing blockers, comparing it between normal and diabetic muscles.

The isolated phrenic nerve-diaphragm muscies in normal (male mice of the $d d r$ strain, weighing 32-44 g) and alloxaninduced diabetic (male mice of the $d d Y$ strain, weighing 21-39 g, with a blood glucose level of $360-628 \mathrm{mg} / \mathrm{dl}$ ) mice were used. Alloxan monohydrate $(85 \mathrm{mg} / \mathrm{kg}$. Nakarai) was injected into the tail vein of a mouse which was used at 28-40 days after the injection. The twitch tensions were elicited by the nerve stimulation $(0.2 \mathrm{~Hz}$, supramaximal, $1 \mathrm{msec}$ ) through a bipolar platinum electrode and were recorded isometrically as previously reported (3). SuCh (Nakarai), decamethonium $\left(\mathrm{C}_{10}\right.$. Sigma) and pancuronium ( $P C$, Sankyo) were applied cumulatively at 2 min-intervals. The inhibitory responses were presented as a percentage of the control value for $1 \mathrm{~min}$ before applying these drugs. $\beta$-Eudesmol was dissolved in propylene glycol at the final concentration of $0.2 \%(\mathrm{~V} / \mathrm{V})$, which showed no effects on twitch response. This concentration of propylene glycol did not affect the action of SuCh or PC, but potentiated that of $\mathrm{C}_{10} 1.5$-fold in normal muscles and 1.7 . fold in diabetic ones (data not shown).

SuCh induced neuromuscular blockade 1.3-fold more potently in diabetic muscles than in normal ones. Pretreatment with 80 / $\mathrm{M} \beta$-eudesmol for $1 \mathrm{hr}$ shifted the concentration-inhibition curve for SuCh to the left by 3.1 -fold in normal muscles and by 6.3 fold in diabetic ones (Fig. 1A). Twenty micromolar concentration of $\beta$-eudesmol shifted the same curve 1.3-fold in normal muscles and 1.5-fold in diabetic ones (data 


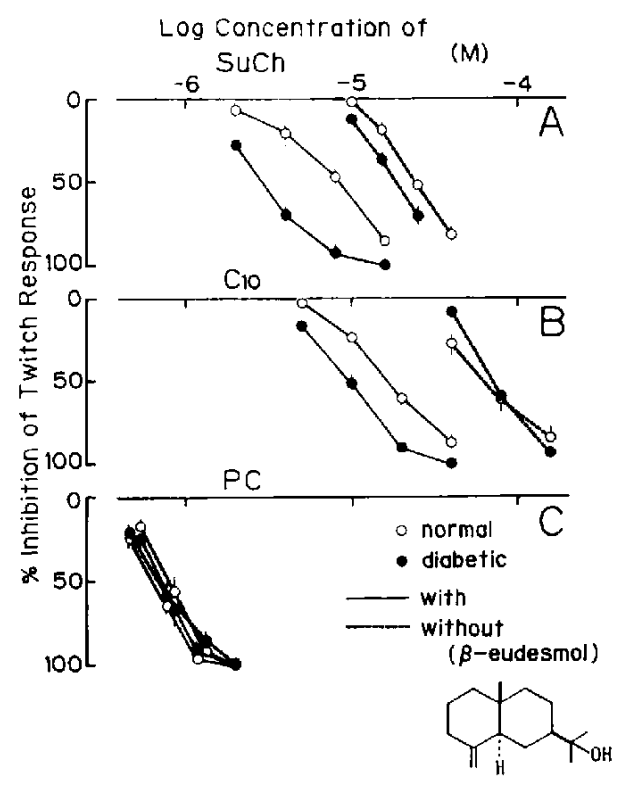

Fig. 1. The potentiating effect of $\beta$-eudesmol on depolarizing neuromuscular blockers in phrenic nerve-diaphragm muscles of mice. The percent inhibitions of nerve-evoked witch responses are plotted against the $\log$ concentration of succinylcholine (SuCh: upper, A), decamethonium ( $\mathrm{C}_{10}$ : middle, $B$ ) and pancuronium ( $P C$ : lower, $C$ ) in normal (O) and alloxan-diabetic (O) mice pretreated with (solid lines) or without (broken lines) $80 \mu \mathrm{M} \beta$-eudesmol for $1 \mathrm{hr}$. The chemical structure of $\beta$-eudesmol is depicted in the lower part of $C$. IC50 (95\% confidence limits, $\mu \mathrm{M}): 24.3$ (22.426.3) (-- --) $18.3(16.9-19.8)^{* *(---)}, 7.81$ $(6.51-9.37)^{++}(-O-) .2 .90 \quad(2.57-3.27)^{* *+1+}$ $(-\mathrm{O})$ in A: $66.0(51.9-83.8) \quad(--\bigcirc--), 75.2$ $(70.8-79.8)(--\cdots) .17 .0(15.2-19.1)^{++}(-\bigcirc-)$. $9.45(8.49-10.5)^{* *+}(-O-)$ in B; $0.744(0.617-$ $0.896) \quad\left(-O^{--}\right), 0.748 \quad(0.660-0.849) \quad(\cdots-\cdots)$. $0.674(0.568-0.800)(-\bigcirc-) .0 .660(0.574-0.759)$ $(-O)$ in $C\left(^{* *}: P<0.01\right.$, compared with the effect in normal mice and +t: $P<0.01$, compared with the effect in the absence of $\beta$-eudesmol by the unpaired t-test or Welch's $t$-test, $n=4-5$ ).

not shown). These concentrations of $\beta$ eudesmol alone showed a little or no effect on twitch tensions. The blocking effect of $C_{10}$ was also potentiated by 80 "M $\beta$-eudesmol. 3.9 -fold in normal muscles and 6.3 -fold in diabetic ones, although the effect of $C_{10}$ without $\beta$-eudesmol was not significantly different in both kinds of muscles (Fig. 1B).
$\beta$-Eudesmol $(80 \mu \mathrm{M})$ did not change the effects of PC (Fig. 1C).

As reported previously, the hypersensitivity to neuromuscular blockers in diabetic muscles was restricted to the depolarizing one but not to the competitive one (2). In the present study, no significant difference in the sensitivity to $\mathrm{C}_{10}$ alone in propylene glycol solution was observed between normal and diabetic mice, but $\mathrm{C}_{10}$ dissolved in water solution was rather less potent in diabetic muscles. Minker et al. (4) also reported that alloxan-diabetic rats were less sensitive to $C_{10}$. although they exhibited hypersensitivity to SuCh. $C_{10}$ does not exhibit the competitive type of blockade and is reported to block the open channel of the nicotinic acetylcholine receptor, but SuCh does not $(5,6)$. Therefore, the difference between SuCh and $\mathrm{C}_{10}$ may be attributed to the different mechanism.

The potentiation effect of $\beta$-eudesmol on SuCh-induced blockade was dependent on the treatment time. The blocking effect of SuCh was not affected by 10 min-treatment with $\beta$-eudesmol in both muscles, but potentiated 2.1 -fold by $30 \mathrm{~min}$ - and 2.8 fold by 60 min-treatment in normal muscles, and it was potentiated 2.8 -fold by $30 \mathrm{~min}$ and 6.3 -fold by 60 min-treatment in diabetic muscles (Fig. 2). This indicates that the potentiating effect of $\beta$-eudesmol became equilibrated by longer treatment and became greater in diabetic muscles.

The high concentration $(200 \mu \mathrm{M})$ of $\beta$ eudesmol alone blocked the nerve-evoked twitching without affecting the muscleevoked one (data not shown). The potentiation by $\beta$-eudesmol was restricted to depolarizing neuromuscular blockers but not to a competitive blocker. These data suggest that the potentiating effect of $\beta$-eudesmol to depolarizing blockers may be caused by interaction with the neuromuscular synapse. However, the precise site of action remains to be clarified. The extent of potentiation by $\beta$ eudesmol was more striking in diabetic muscles than in normal ones. $\beta$-Eudesmol caused more noticeable hypersensitivity to SuCh and some extent of hypersensitivity to $\mathrm{C}_{10}$ in diabetic mice. despite there being no difference in the sensitivity to $C_{10}$ alone. The potentiating effect of $\beta$-eudesmol was satu- 


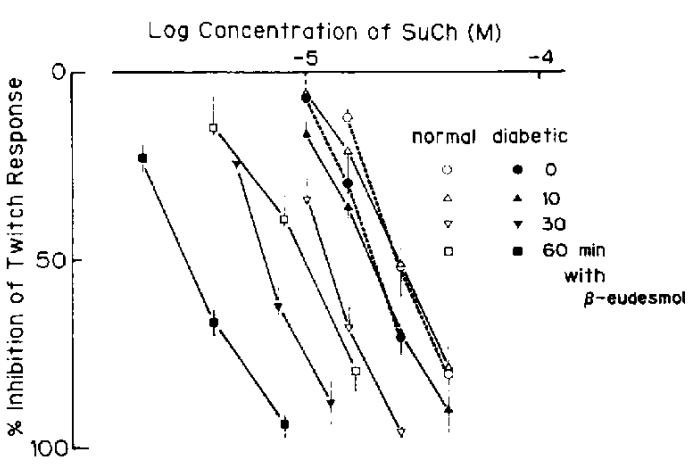

Fig, 2. Influence of pretreatment time on the potentiating effect of $80 \mu \mathrm{M} \beta$-eudesmol on SuCh in phrenic nerve-diaphragm muscles of mice. The percent inhibitions of nerve-evoked twitch responses are plotted for $0 \mathrm{~min}$ (circle), $10 \mathrm{~min}$ (triangle), 30 min (reversed triangle) and $60 \mathrm{~min}$ (square) pretreatment against the log concentration of SuCh in normal (open symbols) and alloxan-diabetic (closed symbols) mice pretreated with (solid lines) or without (broken lines) $\beta$-eudesmol. 1050 ( $95 \%$ confidence limits, $\mu \mathrm{M}): 25.2(22.2-28.7)(0 \mathrm{~min}) .24 .4$ (21.1-28.4) (10 min). $12.1(10.7-13.6)$ (30 min). $8.95(7.43-10.8)(60 \mathrm{~min})$ in normal: $19.3(17.4-$ 21.4) (0 min). $18.2(16.0-20.7)(10 \mathrm{~min}), 6.88$ $(6.21-7.62)(30 \mathrm{~min}), 3.07(2.80-3.36)(60 \mathrm{~min})$ in diabetic muscles $(n=4-7)$.

rated after 30 min-treatment in normal muscles, whereas further potentiation was observed in diabetic muscle after $60 \mathrm{~min}$ treatment. These results suggest that $\beta$ - eudesmol modifies the sensitivity to the depolarizing blockers of diabetic mice into a higher susceptibility.

\section{References}

1 Kimura, M., Kimura, I., Muroi, M., Yoshizaki, M. and Hikino, H.: Pharmacological evidence for an interaction between constituents (blend effect) of the Japanese-Sino medicine "Keishi-kazyutubu-tō" in neuromuscular blockade in diabetic mice. Phytotherapy Res. 1, 107-113 (1987)

2 Kimura, M., Kimura, I., Nojima, H. and Muroi, M.: Diabetes mellitus-induced hypersensitivity of mouse skeletal muscles to acetylcholine and succinylcholine. Japan. J. Pharmacol. 40, 251256 (1986)

3 Kimura, M., Kimura, I., Takahashi, K., Muroi, M., Yoshizaki, M., Kanaoka, M. and Kitagawa, I.: Blocking effects of blended paeoniflorin or its related compounds with glycyrrhizin on neuromuscular junctions in frog and mouse. Japan. J. Pharmacol. 36, 275-282 (1984)

4 Minker, E., Kac, P. and Koltai, M.: Effect of muscle relaxants on the motor endplate of diabetic and glucocorticoid pretreated rats. Acta Physial. Hung. 67, 257-266 (1986)

5 Adams, P.R. and Sakmann, B.: Decamethonium both open and blocks endplate channels. Proc. Natl. Acad. Sci. U.S.A. 75, 2994-2998 (1978)

6 Milne, R.J. and Byrne, J.H.: Effects of hexamethonium and decamethonium on end-plate current parameters. Mol. Pharmacol. 19, 276 $281(1981)$ 